\title{
Soft tissue sarcoma, a multidisciplinary team decision: all experts for one correct management
}

\section{Opinion}

Soft tissue sarcoma (STS) is a rare and aggressive malignancy that can affect the mesenchymal tissues all over the body. The radical oncologic surgery remains the mainstay treatment of the localized disease. Radiation therapy is usually delivered either pre-operatively or in the post-operative setting to control the risks of local recurrence. There are few indications for neo-adjuvant chemotherapy, but once the disease becomes generalized, the only options reside in few lines of chemotherapy. The main objectives of the cytotoxic treatment will be the prolongation of life trying to stabilize the disease progression and to control the patients' symptoms. Despite all the progress of the Medical Oncology, STS wasn't much affected, may be because of its heterogeneity, multiple histologic subtypes, high rates of molecular and genetic mutations responsible for its aggressive behavior. Prognostic factors mainly include the size of the tumor, the histological grade and subtype associated with the possibility of a complete surgical resection. Thus, the best way to control this disease is an adequate care involving the radiologist, the histo-pathologist, the surgeon, the radiation oncologist, the medical oncologist with the early integration of a specialized sarcoma supportive care team. These members will form a multidisciplinary team with an appropriate expertise to make correct team decisions.

STS are quite heterogeneous, grouping many histologic types and subtypes. Besides doing the correct diagnosis, the histo-pathologist must determine the histologic grade of the tumor which will determine the additive treatment managements. Therefore, the pathologists must be experts in this field and very well trained. According to the literature, there has been much discordance when the pathology slides were re-evaluated by an experienced pathologist, with even a diagnosis of wrong benign processes sometimes. The USA and the European countries are creating centralized pathology laboratories for second reading opinions and slides interpretations: a strategy of a national centralization towards international data integration and sharing. This modality had improved the accuracy of the diagnosis which is a major determinant in the management of these tumors.

The radiologists also contribute to the diagnostic work up by evaluating and describing the loco-regional and distant extension of the disease. They also assess the response to the treatment evaluating the disease progression/recurrence according to standardized radiological criteria.

The surgeons play an imminent role in the adequate management of the STS. They are responsible of doing an adequate diagnostic biopsy: the correct way of doing a biopsy without disease seeding in the needle tract and without altering the resection planes. They will also judge the tumor resectability after the examination of the patients and the discussion of their radiologic file. The aim of the surgery must be an oncologic, radical, "en-bloc" resection with negative margins and avoiding a capsular rupture. Nowadays, there are many progresses in the surgical treatment of STS with the possibility of doing reconstructive surgeries allowing a non-mutilating wide
Volume 6 Issue 2 - 2018

\author{
Georges El Hachem \\ Department of Medical Oncology, Institut Jules Bordet, Belgium
}

Correspondence: Georges El Hachem, Department of Medical Oncology. Institut Jules Bordet, Brussels, Belgium, Email George.el.hashem@hotmail.com, georges.elhachem@bordet.be

Received: March 16, 2018| Published: March 22, 2018

resection of the tumor and the microscopically infiltrated surrounding tissues. Furthermore the amputation surgeries are becoming extremely rare, exceptional unless the tumor location and extension don't allow a conservative surgery. After discussing the case with the radiologist, the pathologist, the radiation and medical oncologists of the MDT, many options may be suggested to reduce the risk of undergoing a debilitating surgery: pre-operative radiation therapy or isolated limb perfusion and exceptionally chemotherapy (except for the ewing sarcoma and the osteosarcoma where a pre-operative chemotherapy is mandatory).

The Medical Oncologist assists to the case presentation and judges with the other members the indications and benefits of the cytotoxic treatments. There is also the possibility to include the patients in clinical trials. In the same perspective, the supportive team in STS is involved all over the treatment steps, even in the curative settings. These patients require a specialized rehabilitation program: special physical therapy techniques, occupational psycho-social care and many times an adequate nutritional support, especially in case of intra-abdominal retroperitoneal STS because malnutrition increases the rates of complications. Additionally, they may intervene in the metastatic settings with the instauration of many palliative measures in order to control the symptoms and to improve the quality of life.

Consequently, the multidisciplinary team will join all these experts in a single reunion around a table. They will discuss the standard of cares thoroughly, the best therapeutic options and the evidence based medicine in association with their expertise in the field. Moreover, they may suggest some molecular tests or gene sequencing profiles facilitating and hastening the inclusion of the patients in clinical trials, mainly for the metastatic disease where the options are limited. Many published reviews and retrospective analyses showed that STS managed by a MDT had better results with improvements in recurrence and disease free survivals, as well as overall survival. It also improved the quality of care and the enrollment in clinical trials. 
In conclusion, STS is a rare oncologic entity with many challenging difficulties in the interpretation of the imaging and histology. They are usually characterized by an aggressive behavior requiring complex and intense treatment modalities, justifying the need of a multidisciplinary surgical, clinical and para clinical cooperation. Making an efficient and effective characterization of the tumor cells along with a correct interpretation of the imaging will result in a correct staging, then an adequate radical curative surgery. Clinicians and surgeons should always keep in mind that suboptimal management of the patients affects their survival and is also associated with an increase in the costs of the professional charges. 